\title{
DISONANCIAS PEDAGÓGICAS EN LA RESOLUCIÓN DE PROBLEMAS DE FÍSICA: UNA PROPUESTA PARA SU SUPERACIÓN DE RAÍZ VYGOTSKIANA
}

\author{
NETO, ANTÓNIO J. ${ }^{1}$ y VALENTE, MARIA ODETE ${ }^{2}$ \\ ${ }^{1}$ Departamento de Pedagogia e Educação. Universidade de Évora. Apartado 94, 7000 Évora. Portugal \\ E-mail: aneto@uevora.pt \\ ${ }^{2}$ Departamento de Educação. Faculdade de Ciências da Universidade de Lisboa. 1700 Lisboa. Portugal
}

\begin{abstract}
SUMMARY
Based on Vygotsky's thinking, this paper aims at analysing the number of difficulties students usually experience when trying to cope with problem solving in physics. Both cognitive and metacognitive considerations and affective ones are explored. Some empirical evidence (both quantitative and qualitative), taken from a broad metacognitively oriented field study, is taken into account. The empirical part of this study was carried out in the field of physics, at two Portuguese high schools (tenth grade students), being supported by an experimental versus control design. The analysis of data seems to indicate significantly higher progress for the experimental pupils as compared with their control peers. Our claim that a metacognitively oriented approach might be a suitable means to overcome some of the pedagogical dissonances that typically affect problem solving in physics seems to have acquired considerable support.
\end{abstract}

Education is the acquisition of the art of the utilisation of knowledge. Whitehead

\section{INTRODUCCIÓN}

Como decía Whitehead (1970), la educación con «ideas inertes» no se limita sólo a ser infructuosa; es sobre todo perniciosa: deja por resolver el problema de la necesidad de «mantener vivo el conocimiento», ese conocimiento que puede ser utilizado en la resolución de los problemas con que se enfrenta el educando. Dewey, por otro lado, considera, los problemas, cruciales para la propia actividad del pensamiento: acaban por ser el estímulo o el rastrillo del pensamiento. La naturaleza del problema determina el objetivo del pensamiento, o sea, lo orienta y lo regula. Así se explica que la identificación de la verdadera naturaleza del problema sea considerada, por la mayor parte de los autores, la fase determinante en la resolución de problemas y, de hecho, en la calidad del pensamiento. Es, pues, un momento decisivo de lo que hoy se denomina metacognición, proceso cognitivo que tiene grandes afinidades con el tipo de pensamiento que Dewey destacaba, el pensamiento reflexivo (Brown, 1978, 1987; Flavell, 1987; Valente et. al., 1987).

Una vez afirmada la importancia de éstos y de la resolución de problemas como motor de la actividad del pensamiento, resulta, como corolario, que la actividad educativa escolar debería dedicar una parte significativa del tiempo escolar a este aspecto. Todavía, parece existir una disonancia entre lo que, en este campo, se reclama a la escuela y lo que se supone que la escuela ha de realizar. Superarlo pasa, necesariamente, por garantizar nuevas formas de actuación pedagógica que, en vez de contribuir a «llenar la cabeza de los alumnos de ideas inertes», les puede proporcionar un conocimiento vivo, capaz de ayudarlos a construir un «camino hacia el futuro». 
Como señalaba Vygotsky (1996), la orientación de los objetivos educativos para el desarrollo de tareas mecánicas y rutinarias, o sea, para aquello que el alumno ya sabe realizar, dará lugar a una educación conservadora, poco capaz de servir de motor a ese desarrollo:

El aprendizaje orientado hacia los niveles evolutivos que ya se han alcanzado resulta ineficaz desde el punto de vista del desarrollo total del niño. Este tipo de enseñanza no aspira a un nuevo estadio en el proceso evolutivo, sino que, más bien, va a remolque de dicho proceso. (Vygotsky, en Rivière, 1988, p. 60)

Difícilmente veremos aquí el espejo ideal de lo que debe ser la educación de los niños de hoy para el mundo de mañana. Es en la noción vygotskiana de zona del desarrollo próximo (o potencial) donde esta perspectiva se encuentra bien fundamentada.

A través de esta noción, Vygotsky resume, desde el punto de vista de la enseñanza-aprendizaje, los principios básicos de su teoría psicopedagógica, principalmente el desarrollo de la transformación de un proceso interpersonal (social y cultural) en intrapersonal y el papel de los educadores o de los compañeros más competentes en este proceso. La zona de desarrollo próximo, afirma, «es la distancia entre el nivel real del desarrollo del niño, determinado por su capacidad de resolver un problema independientemente, y el nivel de desarrollo potencial, determinado a través de su capacidad de resolución del problema bien con la ayuda de un adulto o de un compañero más competente (Vygotsky, 1986a, 1986b).

Vygotsky atribuye a la escuela, o sea a la educación sistemática y organizada, el papel fundamental para ayudar al alumno a dar el salto cognitivo hacia el futuro. Una de las herramientas más poderosas que la escuela tiene a su disposición para cumplir esos propósitos son los conceptos científicos -según Vygotsky claramente diferenciados de los conceptos espontáneos-, al hacer corresponder a ambos dos formas distintas, aunque interactivas y sinergéticas, del conocimiento y del pensamiento.

La física, dada la riqueza de sus conceptos y el desafío cognitivo que los mismos pueden implicar, es una asignatura especialmente adecuada para poder crear situaciones reales de clase que se encuadren en un contexto cercano de los alumnos. Pero, en general, no ha conseguido potenciar esa vocación virtual.

Usualmente la enseñanza de la física tiende a presentar situaciones extremas, sobre todo en lo que concierne a la resolución de problemas: por un lado, situaciones demasiado complejas, alejadas de la zona de desarrollo más cercana del alumno, inductoras, por ello, de bloqueos cognitivos y afectivos; y por otro, situaciones problemáticas rutinarias, correspondientes a aquello que el alumno ya sabe realizar.

Ambas situaciones, aunque por razones diferentes, son incapaces de funcionar como estímulo y motor del desa- rrollo. La función potencialmente formativa de la física acaba por ser, así, desaprovechada.

Una nueva didáctica es, pues, necesaria. Sólo ésta puede contribuir al cambio que las circunstancias reclaman; sólo ésta puede contribuir a la resolución del «problema de la resolución de problemas de física», que ha constituido, en el fondo, nuestro trabajo de investigación. La forma que nos hemos propuesto experimentar para abordar la enseñanza de la física pretende fomentar la competencia de los alumnos en la resolución de problemas para romper con algunos condicionantes inhibidores. Su validación nos imponía la necesidad de encontrar respuestas a múltiples preguntas. Las más importantes han sido:

- ¿Favorecerá ese planteamiento el refuerzo de la competencia de los alumnos en la resolución de problemas de física, ya sean de naturaleza cualitativa o cuantitativa?

- ¿Se conseguirá promover de ese modo el desarrollo de importantes estrategias y procedimientos metacognitivos de resolución de problemas?

- ¿Favorecerá tal planteamiento el desarrollo de comportamientos, hábitos y actitudes metacognitivas (experiencias metacognitivas) que potencien estados de resolución más competentes?

- ¿Podrá contribuir a un cambio positivo de actitudes -en general, poco favorables- en relación con la física y la resolución de problemas de física?

Tomando como referencia la obra de Vygotsky -una obra cada vez más valorada en el mundo de la educación-, el estudio global a que este artículo se refiere (Neto, 1995, 1998) tuvo como finalidad contribuir a que las disonancias educativas antes señaladas pudieran ser por lo menos minimizadas. La complejidad y la multidimensionalidad de la resolución de problemas en cuanto a competencia cognitiva no dejarán, sin embargo, de presuponer dificultades. El éxito de los alumnos ante la resolución de problemas de física estará, de este modo, siempre marcado por fuertes contingencias, inherentes a todo acto creativo, del cual la resolución de problemas constituye una importante expresión (Garret, 1987). A este respecto, vale la pena recordar a Vygotsky (1996): «Crear es fuente de júbilo para el hombre: pero acarrea también sufrimientos conocidos con el nombre de torturas de la creación.» (p. 49)

Reflexionar sobre las dificultades de los alumnos en la resolución de problemas de física y ayudar a atenuar la «tortura creativa» que tendrán que superar para resolverlos constituirán los objectivos teóricos y empíricos de este estudio, materializados en una análisis teórico y en propuestas de actuación concreta de cuño necesariamente multidimensional y sinergético. Como dice Vygotsky, «ambas dimensiones, la intelectual y la emocional, resultan por igual necesarias para el acto creador»; o sea, ambos, «sentimiento y pensamiento», «mueven la creación humana». La resolución de problemas por el hombre es una actividad cognitiva compleja y de múltiples 
facetas que pone en acción no sólo capacidades y competencias estrictamente cognitivas, sino también capacidades, competencias y disposiciones típicas de otros dominios psicológicos.

Inspirados en el referencial propuesto por Schoenfeld para el estudio del comportamiento de los alumnos en la resolución de problemas y apoyados en la descripción de Brady (1991), utilizamos en este estudio la siguiente matriz funcional con respecto a los recursos y las variables psicológicas involucradas:

1) Variables afectivas y motivacionales. Al darles prioridad en la discusión, hemos querido asumir una posición deliberadamente disonante de lo que es la práctica corriente: ignorar o escamotear el papel motor de la vertiente afectiva en el aprendizaje y en la resolución de problemas.

2) Recursos cognitivos específicos. Están relacionados con los contenidos, la lógica, la estructura y los procesos característicos del dominio en cuestión.

3) Recursos cognitivos generales. Corresponden a estrategias y técnicas generales en la resolución de problemas -las heurísticas de Polya (1973), por ejemplo-, o sea, a procedimientos que pueden ser utilizados en la resolución de cualquier problema, sin que importe el contenido específico en juego.

4) Recursos cognitivos intrapersonales. En esta categoría inserimos el caso particular de los estilos cognitivos, como variable diferenciadora del comportamiento individual, y el desarrollo cognitivo del alumno, en particular, su nivel de competencia en la realización del pensamiento lógico en sentido piagetiano.

5) Recursos metacognitivos. Son los componentes responsables del control y la gestión de todos los recursos cognitivos, en interacción dialéctica con las variables afectivas y motivacionales; aparecen asociados a toda una diversidad de designaciones (metacognición, autorregulación, automonitorización, control ejecutivo, etc.). Se trata de una categoría de recursos que sólo últimamente ha merecido alguna atención (Brady, 1991; Schoenfeld, 1989), pero que, por ello, ha constituido uno de los focos de este trabajo.

\section{METODOLOGÍA}

Respecto a la investigación en educación, hemos adoptado, en este estudio, una posición metodológica y epistemológica apoyada en la convicción de que, en investigación educativa, el enfoque cuantitativo y cualitativo, al contrario de antagónicos, deben ser complementados. De este modo, a pesar de que la orientación del estudio es preferentemente cuantitativa, fue complementado con importantes técnicas de naturaleza cualitativa (entrevistas y análisis de contenido de los materiales escritos de los alumnos). La vertiente cuantitativa tuvo como base un diseño casi experimental con grupo de control no equivalente, en el cual se han confrontado los planteamientos tradicional (grupo de control) y experimental. Fueron pilares importantes de esta última, los siguientes:

- Una perspectiva constructivista del conocimiento, en que se presenta no sólo desde su acepción estática, sino también dinámica; no sólo como contenido sino, también como proceso.

- Un apoyo sistemático en esquemas de organización del conocimiento. Toda la estrategia experimental fue concebida de modo que la cinemática y la dinámica, en vez de ser tratadas de forma más o menos aislada como es habitual, fuesen abordadas de un modo integrado, potenciando afinidades, paralelismos y analogías.

- Una fuerte orientación cualitativa, no con el objetivo de dar a entender a los alumnos que la física sólo tiene que ver con conceptos, sino en el sentido de minimizar en ellos la idea de que la física tiene que ver sólo con fórmulas.

- Una intensa preocupación metacognitiva, concretada en la explicitación y en el ejercicio de procesos y estrategias de resolución de problemas, y encarada como un elemento de unión de los aspectos anteriores.

\section{La intervención: mecanización versus concienciación}

Caracterizaremos en seguida el método experimental porque tiene especifícamente que ver con la resolución de problemas, confrontándolo con el método tradicional -expresión que, ante una mejor designación, quiere significar, para nosotros, las metodologías habitualmente adoptadas en las clases de física, en los que aquellos principios, por inconvenientes diversos, acaban por no ser puestos en práctica.

\section{Método tradicional}

Tiene como base la utilización de tareas rutinarias, que conducen a comportamientos mecánicos, automáticos y acríticos. Estas tareas son esencialmente ejercicios de computación numérica o «problemas cerrados», casi siempre apoyados en la utilización de reglas y algoritmos anteriormente aprendidos y automáticamente aplicados. Les corresponden enunciados cortos y resumidos, en los que los números predominan sobre las palabras; en los que la situación física es presentada de forma descontextualizada; en los que, al final, todas las atenciones se centralizan en la fórmula. El alumno es estimulado a utilizar y ejercitar este tipo de estrategia. Pasa lo mismo con la diferencia entre lo esencial y lo accesorio, la identificación de la información-clave y la concepción previa de un plano de resolución.

Es muy probable que, para la mayoría de los alumnos, estas actividades se localicen más allá de la respectiva zona de desarrollo próximo. Serán, en este sentido, actividades que poco o nada contribuirán al desarrollo 
efectivo del alumno. Esto no significa que no puedan tener algunas dificultades; ésas serán, sin embargo, sobre todo derivadas de la falta de conocimientos básicos o de la dificultad de desarrollo en la memoria de reglas previamente aprendidas. La mayor parte de las actividades («problemas») propuestas en los manuales didácticos en el final de cada capítulo son de ese tipo. En virtud de haber intentado seguir con el grupo de control el planteamiento preconizado por un manual de este tipo ha sido esta orientación la que esencialmente se aplicó en el grupo.

\section{Método experimental}

Sin negar la importancia de los ejercicios, de las reglas y de los algoritmos, como prerrequisitos necesarios (aunque no suficientes) para la resolución de problemas cuantitativos de física, el planteamiento experimental intentó no quedarse en los prerrequisitos. Se apoyó en tareas problemáticas de nivel más alto, concebidas con la intención deliberada de no viciar al alumno en comportamientos como el «operativismo ciego», la obsesión por la fórmula y por los datos numéricos, el pensamiento mecánico y automático.

Buscando niveles de desarrollo más elevados que los de los abordajes anteriores, las tareas que aquí se utilizarán, aunque intelectualmente más exigentes, intentarán llevar a los alumnos (a cada alumno) más allá de sus capacidades reales o potenciales. Se intentará, en otras palabras, llegar al desarrollo potencial de cada uno de ellos. Se pretendía que la novedad de la situación fuese ahora suficientemente moderada para, por un lado, evitar que el alumno experimentase bloqueos cognitivos y afectivos insuperables; y, por el otro, garantizar un mínimo de conflicto cognitivo, necesario para activar los mecanismos motivacionales (Flavell, 1987).

Como soporte material de este procedimiento, los alumnos tenían a su disposición fichas de resolución metacognitiva de problemas de física. Éstas fueron pensadas con el objetivo de proporcionar un ejercicio sistemático y orientado en estrategias cognitivas y metacognitivas de resolución de problemas, y de incentivar a los alumnos a explicar verbalmente (en este caso, por escrito) los procesos de pensamiento que dan cuerpo a tales estrategias. Estas fichas eran complementadas con otros materiales de apoyo, como textos, documentos informativos o mapas de conceptos. Eran, además, exhaustivamente discutidas en clase -algunas fueron también propuestas como deberes para hacer en casa. La discusión se concretaba de las más diversas maneras: con toda la clase, con los alumnos individualmente o en grupos de dos. Sobre todo nos inspiramos en la técnica de pensar en voz alta a los pares, de Whimbey y Lochead (1986), trabajo que consideramos particularmente favorable en el intento de llevar a los alumnos a expresar oralmente lo que pensaban, siempre con la ayuda de un compañero del grupo.

El manual didáctico aparece aquí como una fuente complementaria de información. Dadas las características renovadoras de este método, no existía en el mercado ningún manual que se adaptase; así el investigador tenía que construir los materiales aplicados.

Importante fue también el recurso a toda una diversidad de problemas de tipo cualitativo. Éstos tienen la ventaja, ante los problemas cuantitativos, que condenan al fracaso cualquier intento del alumno de emplear, como prioridad, el recuerdo obsesivo de la fórmula, en la resolución de problemas.

Así, en vez de los enunciados propuestos por los manuales y adoptados normalmente en el método tradicional, decidimos crear enunciados más adaptados a los grupos experimentales. Al contrario de los primeros, éstos eran enunciados extensos, en los cuales, las palabras predominaban sobre los números (Cuadro I).

Cuadro I

Un problema de enunciado extenso.

\section{PROBLEMA}

Dos amigos, que pesan ambos $75 \mathrm{~kg}$., se encontraban de vacaciones en una playa del Algarve. Uno de ellos tenía un barco de motor de fibra de vidrio, de tecnología japonesa. El barco pesaba (con motor incluido) cerca de $300 \mathrm{~kg}$.

Los dos pasaban la mayor parte del día haciendo pruebas de velocidad con el barco. Controlaban el tiempo que necesitaban para llegar a una boya situada a $0,8 \mathrm{~km}$ de la costa. El mejor tiempo fue de $40 \mathrm{~s}$. Un día, que el mar estaba calmado y soplaba una brisa suave, salieron convencidos de que el récord anterior sería superado. Verificaron, por medio de un velocímetro, que, en los primeros $10 \mathrm{~s}$., la velocidad había subido uniformemente hasta 36 $\mathrm{km} / \mathrm{h}$. Decidieron, por ello, mantener la misma media de velocidad durante todo el recorrido.

Al llegar a la boya, ¿cuál fue la reacción que tuvieron los dos amigos? ¿Satisfacción o desilusión?

Por ser más descriptivos, estos enunciados permiten una mejor contextualización de la situación física. Ese mismo carácter descriptivo implica que, antes de intentar recordar las fórmulas adecuadas, el alumno tenga que utilizar y desenvolver estrategias metacognitivas que tienden a identificar y caracterizar la naturaleza del problema. El alumno se ve así empujado a emprender esfuerzos deliberados, en el sentido de distinguir la información relevante de la información irrelevante, intentando localizar en el enunciado las palabras o expresiones clave (consideradas decisivas como estímulo para activar la memoria). Este tipo de enunciado implica una mayor preocupación al provocar que las estrategias mentales de planificación intervengan, incentivando el recurso de procesos de avaluación y control metacognitivo.

\section{Los sujetos}

Los sujetos que participaron en el estudio eran alumnos del $10^{\circ}$ año que frecuentaban la asignatura de ciencias 
físicoquímicas. La constitución de los grupos no pudo ser realizada aleatoriamente por tratarse de clases ya organizadas, que pertenecían a dos escuelas secundarias (X y Y) de la ciudad de Évora. A efectos de tratamiento estadístico, los hemos dividido en tres grupos: dos experimentales (E1 y E2) y uno de control (C). Les correspondía, respectivamente, un planteamiento experimental y uno de tipo tradicional. Los grupos Cy E1 frecuentaban la escuela secundaria $X$; la función docente fue, en ambos, garantizada por el propio investigador (pero sólo en lo que a física se refiere), durante cinco meses. El grupo C, con media de edades de 15,4 años, estaba constituido por 24 alumnos (15 chicas y 9 chicos); el grupo E1 (media de edades de 16,3 años) estaba, a su vez, constituido por 27 alumnos (16 chicas y 11 chicos). A los dos grupos correspondía un perfil socioeconómico de clase media, perteneciendo cerca de la mitad de sus componentes a comunidades rurales de la zona de Évora.

El grupo E2 se puede considerar un grupo intacto sólo a efectos de tratamiento estadístico. Le correspondían, en efecto, dos subgrupos que provenían de dos clases de la escuela Y. En las dos clases, y durante todo el año escolar, la responsabilidad de la docencia estuvo a cargo de las profesoras respectivas -dos buenas profesoras en aquella escuela, con algunos años de experiencia. Varias dificultades, algunas asociadas a la organización específica de la escuela, impidieron una recogida de datos similares a la de los otros grupos y también hubo menor tiempo de intervención (dos semanas menos). Este grupo se componía de 25 alumnos (13 chicas y 12 chicos, con una media de edades de 15,2 años), residentes la mayoría en la ciudad de Évora y perteneciendo a familias de clase media-urbana.

\section{Instrumentos utilizados y correspondientes variables}

Con la finalidad de organizar mejor toda la diversidad de instrumentos y datos, decidimos considerar, genéricamente, tres campos de variables cuantitativas:

1) Campo nuclear de la resolución de problemas. Constituye el núcleo fundamental de la pesquisa. Está configurado por una serie de procesos, estrategias y estilos directamente relacionados con la competencia de los alumnos en la resolución de problemas de física, sean cuantitativos o cualitativos, y que tienen en común procesos cognitivos o metacognitivos. Les correspondían los siguientes instrumentos de evaluación: Test de resolución de problemas cuantitativos de física, con una versión pretest y otra postest (TRPCTpre, TRPCTpos); Test de resolución de problemas cualitativos de física (TRPCLpre, TRPCLpos); Test de estrategias metacognitivas de resolución de problemas de física (TESTMpre, TESTMpos); Test de comprensión y aplicación de conocimientos de fúsica de $9^{\circ}$ año (TFIS9); Test de comprensión y aplicación de conocimientos de mecánica de $10^{\circ}$ año (TMEC10); Test de pensamiento lógico (TPLOG); e Inventario de estilos epistémicos.

2) Campo de la experiencia metacognitiva. No se trata sólo de procesos propiamente dichos; corresponde a estados y comportamientos del tipo actitudinal que pueden influir en la forma cómo el alumno aborda, ejecuta, controla y evalúa su actividad cognitiva. Está constituido por un complejo de indicadores metacognitivos que consubstancian el instrumento que denominamos Cuestionario de experiencias metacognitivas en la resolución de problemas de física (CEXPM). El Test de estrategias metacognitivas de resolución de problemas de física descrito anteriormente estaba destinado a lo que Flavell denominaba el conocimiento metacognitivo y, dentro del mismo, al conocimiento de tipo procesual. En el caso concreto de este cuestionario, la tipología de la mayor parte de sus ítems encajaba mejor en lo que en el mismo modelo de Flavell aparece con el nombre de experiencias metacognitivas. En este complejo de ítems, esencialmente se contemplan las siguientes experiencias metacognitivas: nivel de comprensión de la tarea problemática en cuestión, antes y después de su resolución afectiva (respectivamente, COMPa y COMPd); nivel de dificultad previo y el experimentado (DIFICa y DIFICd); grado de confianza en relación con la previsión que hacen los estudiantes sobre su capacidad para resolver o no el problema $(\mathrm{CONFa})$ y en relación con la valoración que hacen después de resolverlo sobre su capacidad real (CONFd); nivel de desarrollo y voluntad en la tarea (EMP). Además de ítems de este tipo, han sido incluidos otros dos que constituyen una simbiosis de experiencias metacognitivas con las estrategias metacognitivas de revisión (REV) y de evaluación (AVAL) del procedimiento mental.

3) Campo afectivo. Estaba esencialmente asociado a dos tipos de variables: actitud respecto a la resolución de problemas de física y actitud respecto a las ciencias físicoquímicas. Les correspondían, respectivamente, un cuestionario basado en las escalas de Likert, denominado Grado de satisfacción con las ciencias físicoquímicas (SATFQ) y un instrumento ausente en la técnica del diferencial semántico, con el título de Test de actitud para con la resolución de problemas de física (ATRPF).

A excepción del Inventario de Estilos Epistémicos, los otros instrumentos han sido concebidos, adaptados o recopilados por el propio investigador. Se imponía, pues, analizar previamente todo lo que se diga respecto a cuestiones, ya sea relacionadas con la validez de contenido, ya sea con su funcionalidad. En lo que se refiere a la validez del contenido, se omitieron los mismos a la apreciación crítica de un panel de jueces, constituido por dos doctores y un maestro en el área de la educación en ciencias, un maestro en el área de la psicología de la educación y dos profesores de físicaquímica de la enseñanza secundaria, con experiencia profesional.

Una vez introducidas las rectificaciones sugeridas por el referido panel, esos instrumentos han sido objeto de un estudio piloto, realizado en dos clases también de $10^{\circ}$ año de escolaridad de la zona escolar de Évora. Fueron así reveladas algunas insuficiencias antes no previstas o no detectadas, principalmente en referencia a la comprensión por parte de los alumnos del significado de las cuestiones y de las tareas propuestas, y de la operaciona- 
lidad temporal de cada uno de los instrumentos. De acuerdo con los datos obtenidos, se ha procedido, además, a la determinación de indicadores estadísticos apropiados, susceptibles de complementar la información respecto a la calidad de los instrumentos de medida. Intentando evaluar la consistencia interna de los conjuntos de medidas así proporcionadas, se determinó para cada uno de ellos el respectivo coeficiente de fiabilidad (en este caso el $\alpha$ de Cronbach). Se obtuvo, en general, valores bastante aceptables o muy buenos (como es el caso de los tests incluidos en el campo afectivo), con la particularidad (comprensible) de que los pretests revelaron sistemáticamente una calidad inferior a los postests correspondientes. Ésa fue una de las razones que nos llevaron a optar por el modelo de análisis de covarianza como soporte nuclear de la exploración de los cambios cuantitativos observados.

Para reforzar la confianza en los datos y en los resultados obtenidos, haciendo una interpretación más consistente y más fundamentada de los mismos, se procedió igualmente a un análisis de ítems exhaustivo, con determinación de los respectivos índices de dificultad y de discriminación, cuya descripción, por razones de espacio, no puede aquí ser presentada.

Como Cook y Campbell (1979) mantienen, el simple «análisis de las diferencias entre los pretests y los postests de una determinada variable es, muchas veces, una opción inapropiada» (p. 104). Pensamos que ese aspecto era el más interesante de este trabajo: la calidad de los pretests era en general inferior a los respectivos postests. Lo ideal sería disponer de métodos de análisis cuyo algoritmo de procesamiento no funcionase sobre la base del cálculo de diferencias pretest/postest, a pesar de garantizar que el estado inicial de los sujetos se tuviese en cuenta. Verificamos, pues, que el modelo estadístico de Análisis de covarianza-simple (ANCOVA) o multivariada (MANCOVA) - cumplía ese requisito. Fue por ello que ese modelo, así como el correspondiente modelo de Análisis de la varianza, acabaron por constituir la base del tratamiento estadístico de los datos cuantitativos, conforme al esquema ilustrado en el cuadro II.

\section{RESULTADOS}

\section{Adhesión de los alumnos al método de resolución de problemas}

Por razones ya suficientemente expuestas, decidimos en este estudio optar por situaciones problemáticas intermedias entre los problemas académicos típicos y los problemas de la vida real. Estábamos convencidos de que, además de ser más adecuados a las exigencias antes referidas, esos problemas se revelarían, por esa misma razón, más «realistas» que los de la vida real, a pesar de ser menos susceptibles de incentivar el desarrollo del pensamiento crítico de los alumnos y de su metacognición.

La evidencia acabó por darnos la razón, considerando el impacto claramente positivo que esos problemas tuvie-
Cuadro II

Codificación de las variables cuantitativas ajustadas al modelo ANCOVA

\begin{tabular}{lcc}
\hline & Variables dependientes & Covariables \\
Campo nuclear & TRPCTpost & TRPCTpre \\
de resolución & TESTMpost & TESTMpre \\
de problemas & TRPCLpost & TRPCLpre \\
& TMEC10 & TFIS9 \\
& & TPLOG \\
& & \\
Campo de la & Todos los & Todos los \\
experiencia & indicadores post & indicadores pre \\
metacognitiva & correspondientes a & correndientes \\
& este campo & a este campo \\
Campo afectivo & SATpost & \\
& ATRPFpost & SATpre \\
& & ATRPFpre
\end{tabular}

ron en los alumnos de los grupos experimentales. Éstos no parecen haber experimentado dificultades inexorables como lo comprueban sus opiniones expresas a través de entrevistas. Este impacto positivo ha tenido reflejos correlativos en la adhesión de estos alumnos a diferentes vectores del planteamiento implementado, sobre todo en los dos siguientes:

a) método didáctico de resolución de problemas preconizado -método sistemático de orientación metacognitiva, más explícito y más verbalizado que los métodos habitualmente utilizados;

b) enunciados de los problemas-enunciados extensos y descriptivos, en vez de enunciados cortos y resumidos.

\section{Método de resolución de problemas}

Es particularmente importante, por la comparación que proporciona, la opinión de los alumnos que antes habían suspendido (eran muchos en el grupo E1), en lo que respecta al impacto que el referido método ha tenido en ellos:

E. -Después de haber hecho también el año pasado el décimo curso (15 años), ¿te has dado cuenta de las diferencias entre el método de resolución de problemas de este año y del año anterior?

B. -Creo que sí. Hubo una diferencia.

E. $-¿$ En que sentido?

B. -Es que este año... primero se entendía bien el problema.

E. $-i Y$ después?

B. -Después se pasaba a la fórmula.

E. $-¿$ Y el año pasado?

B. - ¡No era así! (Riéndose abiertamente.)

(Alumno del grupo E1 repetidor) 
Comparando el método del año anterior («método tradicional») con el método actual (experimental), el alumno tiene conciencia de la diferencia principal entre ambos, inclinándose en favor del segundo: pensar primero en la situación y sólo después pasar a la fórmula. No dudamos en afirmarlo: en esa sensibilización de los alumnos hacia el papel decisivo de la comprensión cualitativa y de la representación inicial del problema (Chi, Feltovich y Glaser, 1981; Hayes, 1987; Heller y Reif, 1984), residirá uno de los aspectos que mayor éxito habrá conseguido en esta experiencia. Los alumnos al final eran sensibles a una metodología que, al contrario de la del año precedente, les exigía una intervención más activa en el proceso de resolución de problemas, y que implicaba para ello la necesidad de que tomasen decisiones que, en el caso anterior, ya habían sido tomadas. Valoraban, de hecho, el problema: «se presenta de manera diferente», con «cosas del día a día»; esa manera los llevaba a «sacar provecho del problema», «retirar datos», «ver las expresiones más importantes»y «sólo después calcular el problema».

\section{Problemas con enunciados extensos}

Estos enunciados son preferidos frente a los tradicionales, como lo ilustra el siguiente apartado de una entrevista, también con un alumno del grupo E1, que también repetía:

E. -¿Cómo caracterizas los problemas que encuentras en el libro?

S. - ¡Son horribles! (Riéndose con sinceridad.)

E. - ¿En qué distingues los problemas que utilizamos con relación a los del libro?

S. -No sé... Los encuentro más concretos y más fáciles de entender.

E. $-¿$ Con un enunciado tan largo no tenías miedo de confundirte?

S. -Si los leemos con atención es fácil. Fácil... es decir, más fácil.

E. -¿Qué vicio pueden acarrear los problemas del libro a los alumnos?

S. -La dificultad de ser sólo aquello y aquello y aquello; los nuestros nos dejan ver mejor, con más claridad, el tipo de problema. Son más lógicos, nos dan más que pensar. En los otros, nos limitamos a memorizar la fórmula y aplicarla.

El párrafo anterior es de por sí ilustrativo: en pocas palabras, el alumno casi resume los aspectos más importantes de este tipo de enunciados. La aceptación de los alumnos de los grupos experimentales a estos enunciados es una de las conclusiones que en este estudio son puestas en evidencia.

\section{Impacto cuantificado del método experimental}

- El método experimental parece haber contribuido a suavizar las habituales dificultades afectivas sentidas por las alumnos en el estudio de la física y en la resolución de problemas de física.
En el cuadro III se puede verificar que los cambios relativos presentados por los grupos experimentales en el campo afectivo han sido significativamente más favorables que los manifestados por el grupo de control.

Cuadro III

Cambios relativos: síntesis del campo afectivo.

\begin{tabular}{cll}
\hline VARIABLE & $E 1 \propto C$ & $E 2 \propto C$ \\
SATFQ & $\mathrm{E} 1>\mathrm{C}$ & $\mathrm{E} 2>\mathrm{C}$ \\
$A T R P F$ & $\mathrm{E} 1>\mathrm{C}$ & $\mathrm{E} 2>\mathrm{C}$
\end{tabular}

Nota: Al símbolo > corresponde un resultado significativamente superior del primer grupo frente al segundo $(\mathrm{p}<0,05)$; al símbolo $=$ no corresponde ninguna diferencia significativa entre ellos.

Hay que reconocer, sin embargo, que esta diferenciación relativa no se debe tanto al entusiamo creciente de los grupos experimentales por las clases de física o por la resolución de problemas de física, con relación al que habían manifestado en el principio, sino, sobre todo, a un descenso relativamente pronunciado del interés del grupo control. Este descenso fue significativo en ambas variables, siendo mucho más significativo en el caso de SATFQ.

Es decir, parece incuestionable la influencia negativa del método tradicional, frente al experimental, que si bien no ha fomentado en los alumnos el interés por la física (y por la resolución de problemas de física), por lo menos ha evitado que haya disminuido. Admitiendo esa ventaja del método experimental en la dimensión afectiva, tal vez podamos así ver aquí señales inequívocas a favor de los argumentos de que los aspectos afectivos son tanto o más importantes que los cognitivos en el aprendizaje y, obviamente, en la resolución de problemas (Claxton, 1989; Hodson y Reid, 1998; Resnick, 1989). Además, como decía Claxton, «la cognición poco importa cuando se está asustado, deprimido o aburrido» (p. 155).

- El método experimental parece haber favorecido el desarrollo de experiencias metacognitivas potenciadoras de estados de resolución más competentes.

Ciertamente, desde un punto de vista global, el grupo E1, que de entrada no se distinguía estadísticamente de $\mathrm{C}$ en este campo, cambia significativamente frente al de control. Sube con regularidad con relación a sí mismo en todos los indicadores, al tiempo que el de control sube en unos indicadores y baja en otros, siendo en algunos casos este descenso bastante significativo. Eso se traduce en mudanzas relativas significativas en más del $50 \%$ de los indicadores escogidos, conforme lo ilustra el siguiente cuadro-síntesis (Cuadro IV).

- El método experimental parece haber contribuido al refuerzo de la competencia cognitiva de los alumnos en 
Cuadro IV

Mudanzas relativas: Síntesis del campo de la experiencia metacognitiva.

$\begin{array}{ll}\text { VARIABLE } & E 1 \propto C \\ \text { COMPa } & \text { E1 }=\mathrm{C} \\ \text { COMPd } & \text { E1 }>\text { C } \\ \text { FACa } & \text { E1 }>\text { C } \\ \text { FACd } & \text { E1 }>\text { C } \\ \text { CONFa } & \text { E1 }=\mathrm{C} \\ \text { CONFd } & \text { E1 }>\text { C } \\ \text { SEG } & \text { E1 }>\text { C } \\ \text { ATEN } & \text { E1 }=\mathrm{C} \\ \text { REV } & \text { E1 }=\mathrm{C} \\ \text { EMP } & \text { E1 }=\mathrm{C} \\ \boldsymbol{A V A L} & \mathrm{E} 1>\mathrm{C}\end{array}$

la resolución de problemas de física, sobre todo en los de naturaleza cuantitativa.

En referencia a la competencia cognitiva de los alumnos en la resolución de problemas de física, verificamos inicialmente que el grupo E1 era, de los tres, el que parecía globalmente menos preparado. Pero, teniendo en cuenta la información del cuadro $\mathrm{V}$, concluimos que fue éste el grupo que, al parecer, más se ha beneficiado con la experiencia. Ultrapasa claramente el grupo de control, aconteciendo eso en ambas de las variables en consideración, con especial incidencia en el caso de TRPCT.

\section{Cuadro V}

Mudanzas relativas: Síntesis de la competencia cuantitativa y cualitativa.

\begin{tabular}{lll}
\hline VARIABLE & $E 1 \propto C$ & E2 $\propto C$ \\
& & \\
TRPCT & E1 $>\mathrm{C}$ & E2 $>\mathrm{C}$ \\
TRPCL & $\mathrm{E} 1>\mathrm{C}$ & $\mathrm{E} 2=\mathrm{C}$ \\
\hline
\end{tabular}

El grupo E1 ha presentado en este componente un progreso significativo considerable (pasa de 38,2 a 76,8, mientras que $\mathrm{C}$ baja de 46,5 a 32 ). Este será el aspecto en que la experiencia habrá conseguido resultados más visibles. También el grupo E2 ha obtenido aquí su mejor resultado relativo; de 46,8 a 74, en TRPCT) .

En relación con los problemas cualitativos, la experiencia continúa haciendo subir los grupos E1 y E2. La subida de E1 es bastante considerable; E2 apenas sube «casi significativamente» ( $\mathrm{p}<0,10)$; en términos de comparación relativa, se obtienen ganancias estadísticamente idénticas a las del grupo de control.
- Aparentemente, fue menos acentuado el éxito relativo del método experimental en la promoción del desarrollo de estrategia y procedimientos metacognitivos de la resolución de problemas.

Teniendo en cuenta los cambios, los resultados apuntan de esta vez a una subida significativa de E1 y a una subida menos significativa de E2 (Figura 1). Los progresos evidenciados son además diferenciados, apreciando el conjunto de los indicadores en cuestión. En efecto, emergen nítidamente del gráfico dos subconjuntos de indicadores, siendo aplicable a E1, pero también a los otros dos grupos. En el primer subconjunto, los alumnos obtienen (tanto al principio como al final) resultados visiblemente más elevados, relativamente a los obtenidos en el segundo). Los dos subconjuntos deberán configurar procesos metacognitivos respectivamente diferentes; más simples y menos amplios en el primer caso; más complejos y menos amplios en el segundo. De éstos se destacan los indicadores PREVISIÓN y PLAN que, como es sabido, constituyen dos macroestrategias fundamentales en el modelo de Brown (1978) y dos importantes metacomponentes en el modelo de Sternberg (1986). Les corresponden, de este modo, estrategias metacognitivas de nivel jerárquicamente más elevado, las cuales exigen la movilización de todo un abanico de procesos de pensamiento de nivel inferior (componentes) y que, por ello, exigen mayor nivel de explicitación y un correlativo tiempo mayor de intervención para que los progresos puedan resultar visibles.

Existen además algunos indicios de que el grupo E1 pueda, a pesar de todo, haber conseguido mayor éxito relativo en el desenvolvimiento de este tipo de estrategias. Basta con destacar mejor la aportación de sus representantes en la resolución oral verbalizada del problema que era propuesto en las entrevistas, así como una aparente mayor concienciación de los pasos de la resolución de problemas y de las dificultades inherentes, detectada también en las entrevistas. Esto puede deberse al mayor tiempo de intervención de que fue posible disponer para este grupo, en concreto para E2 y también al hecho de haber sido orientado por el propio investigador. Por razones comprensibles, es siempre difícil (tal vez imposible) para un investigador conseguir comunicar totalmente sus modelos a todos aquéllos que trabajan con él. Ahí reside una de las principales dificultades en la interpretación de los resultados de gran parte de la investigación educacional en la realidad compleja y sistemática de la clase. Ese menor impacto relativo de la experiencia para el caso del grupo E2 fue, además, con mayor o menor incidencia, una constante en todos los campos que hemos analizado.

\section{CONCLUSIÓN}

Juzgamos pertinente esbozar ahora una breve síntesis de la reflexión que, a lo largo del estudio global, intentamos realizar respecto a lo que es la realidad de las clases de física, en la mayoría de nuestras escuelas. Intentando hacer a una radiografía completa de la situación, hemos 


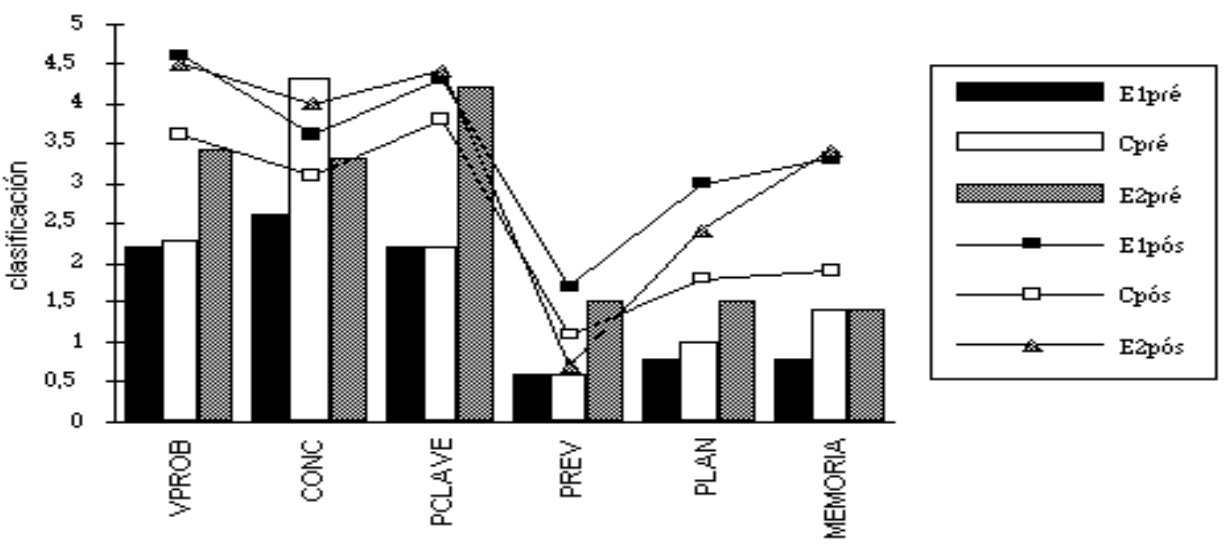

VPROB: Identificación del verdadero problema. CONC: Identificación de los principales conceptos físicos.PCLAVE: Identificación de las palabras o expresiones que son la clave para la resolución del problema. PREV: Formulación de una previsión para la respuesta al problema. PLAN: Explicitación de un plan previo de resolución. MEMORIA: Explicitación de los principales conocimientos de física que fue necesario buscar en la memoria a largo plazo.

destacado aspectos como: la opción por «metodologías de la superficialidad»; el recurso a métodos de resolución de problemas rutinarios y asistemáticos, omitiendo el papel decisivo de la organización del conocimiento; el recurso al «operativismo ciego», con el consecuente primado de la fórmula y de la parte teórico-cuantitativa; el énfasis excesivo en ejercicios y problemas-tipo; el dominio del conocimiento declarativo y la consecuente falta de consideración y explicitación del conocimiento procesual; la omisión o consideración indebida del conocimiento espontáneo de los alumnos; la total preponderancia de la vertiente lógico-formal del pensamiento, con la consecuente falta de consideración del pensamiento creativo; la uniformidad y homogeneidad de los procedimientos didácticos utilizados, ignorando toda la diversidad de estilos cognitivos y motivacionales presentes en una clase.

Esta situación impone la necesidad de cambios pedagógicos y metodológicos (Gil Pérez y Martínez Torregrosa, 1983), capaces de romper con el pasado, con aquello que en el pasado funcione como inhibidor del futuro. Se presuponen, al final, nuevas perspectivas, nuevos cuadros pedagógicos y didácticos, los cuales no pueden dejar de tenerse en cuenta:

a) la opción por estrategias sistémicas y profundas, en vez de estrategias fragmentadas y superficiales;

b) el refuerzo de la dimensión cualitativa del conocimiento, sin invalidar la importancia de la dimensión cuantitativa; al contrario, valorizándola;

c) la importancia del conocimiento procesual, sin negar la importancia equivalente del conocimiento declarativo; d) la necesidad de evitar el «operativismo ciego», a través de la realización de análisis cualitativos iniciales amplios, con la subsiguiente construcción de representaciones (internas y externas) consistentes y productivas;

e) la importancia conferida a la explicitación (con concienciación) del conocimiento, con las implicaciones consecuentes en términos de desarrollo de comportamientos metacognitivos;

f) el reconocimiento del papel decisivo desempeñado por la organización del conocimiento en la resolución de problemas.

El cambio pedagógico que aquí preconizamos para la enseñanza y para la resolución de problemas de física no constituye para nosotros una mera utopía. El sentido favorable de los resultados del estudio a que se refiere este artículo nos enseña, en efecto, que puede ser viable. Pero muestra también que, a pesar de ser potencialmente viable, su interpretación está marcada por múltiples contingencias: unas de tipo estructural y de organización; otras íntimamente asociadas al resultado de aquéllos que tendrán que ser considerados como los verdaderos promotores de la mudanza: los profesores. El menor impacto de la experiencia en las clases de las profesoras que colaboraron con nosotros es una señal no irrelevante de lo afirmado anteriormente.

La superación (o minimización) de las disonancias pedagógicas que afectan a la enseñanza de la física requiere, todavía, una fuerte puesta al día en la formación de profesores, ya que no pueden ser considerados como simples transmisores de información acabada, sino como auténticos promotores del desarrollo, que tienen como finalidad conseguir un ciudadano científicamente 
educado, en la acepción amplia que este concepto va tomando. Se buscan, en otras palabras, profesores de física diferentes, capaces de asegurar una enseñanza de calidad. Cualquier enseñanza sólo puede ser de calidad en la medida exacta en que el profesor sea capaz de proporcionar a los alumnos ayuda adecuada y oportuna, con especial atención a las tareas que correspondan a las respectivas zonas de desarrollo potencial. Éste es, precisamente, el mensaje de las siguientes palabras de Vygotsky (citado por Gallimore y Tharp, 1990):

«[Teaching is good only when it] awakens and rouses to life those functions which are in a stage of maturing, which lie in the zone of proximal development.» (p. 200)

\section{REFERENCIAS BIBLIOGRÁFICAS}

BRADY, R.R. (1991). A close look at student problem solving and the teaching of mathematics: predicaments and possibilities. School Science and Mathematics, 91(4), pp. 144-151.

BROWN, A. L. (1978). Knowing when, where and how to remember: a problem of metacognition, en Glaser, R. (ed.). Advances in instructional psychology. Hillsdale, Nueva Jersey: Lawrence Erlbaum Associates.

BRUNER, J. (1966). Toward a theory of instruction. Cambridge, Mass: Harvard Press.

CHI, M.T.H., FELTOVICH, P.J. y GLASER, R. (1981). Categorization and representation of physics problems by experts and novices. Cognitive Sience, 5, pp. 121-152.

CLAXTON, G. L. (1989). Cognition doesn't matter if you are scared, depressed or bored, en Adey, P. et al. (eds.).Adolescent development and school science. Londres: The Falmer Press.

DEWEY, J. (1933). How we think. Lexington, Mass: Heath and Company.

FLAVELL, J. H. (1987). Speculations about the nature and development of metacognition, en Weinert, F.E. y Kluwe, R.H. (eds.). Metacognition, motivation and understanding. Hillsdale, Nueva Jersey: Lawrence Erlbaum Associates.

GALLIMORE, R. y THARP, R. (1990). Teaching mind in society: Teaching, schooling, and literate discourse, en Moll, L.C. (ed.). Vygotsky and education. Cambridge, Mass: Cambridge University Press.

GARRETT, R.M. (1987). Issues in science education: problemsolving, creativity and originality. International Journal of Science Education, 9(2), pp. 125-137.

GIL PÉREZ, D. y MARTÍNEZ TORREGROSA, J. (1983). A model for problem solving in accordance with scientific methodology. European Journal of Science Education, 54(4), pp. 448-457.

HAYES, J.R. (1987). The complete problem solver. Hillsdale, Nueva Jersey: Lawrence Erlbaum Associates.

HELLER, J.I. y REIF, F. (1984). Prescribing effective human problem solving processes: problem description in physics. Cognition and Instruction, 1(2), pp. 177-216.
Un comportamiento competente, consciente y reflexivo del profesor y su capacidad de hacer que los alumnos puedan interiorizar ese tipo de comportamiento constituyen, en este sentido, aspectos básicos de la teoría pedagógica de Vygotsky.

Bruner (1966), continuando en esta línea, afirma:

«Instruction is, after all, an effort to assist or to shape growth.» (p. 1)

Todo ello con la hipótesis de que aquello que el alumno sea capaz de realizar hoy en colaboración con el profesor podrá realizarlo solo en el futuro (Vygotsky, 1986b, p. 188).

HODSON, D. y REID, D. (1988). Changing priorities in science education. School Science Review, 70, pp. 101-108.

NETO, A.J. (1995). «Contributos para uma nova didáctica da resolução de problemas: um estudo de orientação metacognitiva en aulas de física do ensino secundário.» Évora: Universidad de Évora. Tesis de doctorado.

NETO, A. J. (1998). Resolução de problemas en física: conceitos, processos e novas abordagens. Lisboa: Instituto de Inovação Educacional.

POLYA, G. (1973). How to solve it (2a. ed.). Hillsdale, Nueva Jersey: Princeton University Press.

RESNICK, L.B. (1989). Education and learning to think. Washington, DC: National Academy Press.

RIVIÈRE, A. (1988). La psicologia de Vygotski (3a. ed.). Madrid: Visor.

SCHOENFELD, A.H. (1989). Teaching mathematical thinking and problem solving, en Resnick, L. y Klopfer, L. (eds.). Toward the thinking curriculum: current cognitive research. Washington, DC: ASCD.

STERNBERG, R.J. (1986). Intelligence applied. San Diego: Harcourt, Bruce, Jovanovitch.

VALENTE, M.O., GASPAR, A., SALEMA, M.H., MORAIS, M.M. y CRUZ, M.N. (1987). Aprender a pensar. Lisboa: Departamento de Educação da FCUL, Projecto Dianoia.

VYGOTSKY, L. (1986a). El desarrollo de los processos psicológicos superiores. Barcelona: Crítica.

VYGOTSKY, L. (1986b). Thought and language. Cambridge, Mass.: The MIT Press.

VYGOTSKY,L.(1996). La imaginación yel arte en la infancia (3a. ed.). Madrid: Ediciones Akal.

WHIMBEY, A. y LOCHHEAD, J. (1986). Problem solving and comprehension (4a.ed.). Hillsdale, Nueva Jersey:Lawrence Erlbaum Associates.

WHITEHEAD, A. N. (1970). The aims of education. Londres: Ernest Benn Limited. 